ADVERSE DRUG REACTION

\title{
Delayed reactivation of haloperidol induced photosensitive dermatitis by methotrexate
}

\author{
G P Thami, S Kaur, A J Kanwar
}

Postgrad Med J 2002;78:1 16-117

A man whose psoriasis was well controlled on methotrexate treatment developed pellagra-like photosensitive dermatitis when he started taking haloperidol. Reactivation of his dermatitis was observed with methotrexate as a recall photosensitivity phenomenon. This false photosensitivity reaction of methotrexate is an important but rarely encountered adverse effect. A possible interaction between methotrexate and haloperidol is emphasised.

M ethotrexate has been associated with a variety of cutaneous adverse reactions, of which recall or reactivation of acute sunburn response is a peculiar reaction. This has also been termed as a false photosensitivity reaction. ${ }^{1-4}$ Methotrexate is also well known for its interaction with other drugs. Pellagra-like dermatitis, an adverse reaction of many cytotoxic drugs like 6-mercaptopurine, 5-fluorouracil, and azathioprine, however, has not been reported with the use of methotrexate..$^{5}$ Although phenothiazines are commonly associated with photosensitivity, haloperidol, a closely related drug, has rarely been reported to induce photosensitivity. ${ }^{6}$

\section{CASE REPORT}

A 41 year old man had been taking weekly oral methotrexate ( $15 \mathrm{mg}$ ) for the past 10 months for psoriasis. Haloperidol 1.5 mg twice daily was then introduced for his psychotic illness. Two weeks after staring haloperidol, he presented with a sudden onset of redness and swelling of face and hands accompanied by redness and watering of both eyes. There were no other complaints, his food intake was normal, and he did not take alcohol or herbal medicines. He denied having excessive exposure to the sun in the recent past. His general physical and systemic examination was normal. His psoriasis was well controlled and he was applying emollients over a few well defined plaques of psoriasis.

Examination revealed diffuse erythema, oedema, scaling, and erosions over his face, anterior aspect of the neck, and dorsa of both hands ( fig 1). It was sharply demarcated to photoexposed areas with characteristic sparing of upper eyelid folds, retroauricular and submental areas. In addition, he had exfoliative cheilitis, angular cheilitis, diffuse oral mucositis, bilateral conjunctival injection, photophobia, and excessive lacrimation. A differential diagnosis of pellagra-like dermatitis precipitated by haloperidol, photosensitivity to haloperidol, exacerbation of psoriasis, and a possible interaction of haloperidol and methotrexate leading to photosensitive dermatitis was considered.

The patient was hospitalised and investigations revealed a normal complete blood count, platelet count, urine analysis, and hepatic and renal function tests. A skin biopsy from the dorsum of the hand revealed parakeratosis, acanthosis, spongiosis, focal epidermal cell degeneration and dermal oedema, accompanied by a moderate lymphomononuclear infiltrate

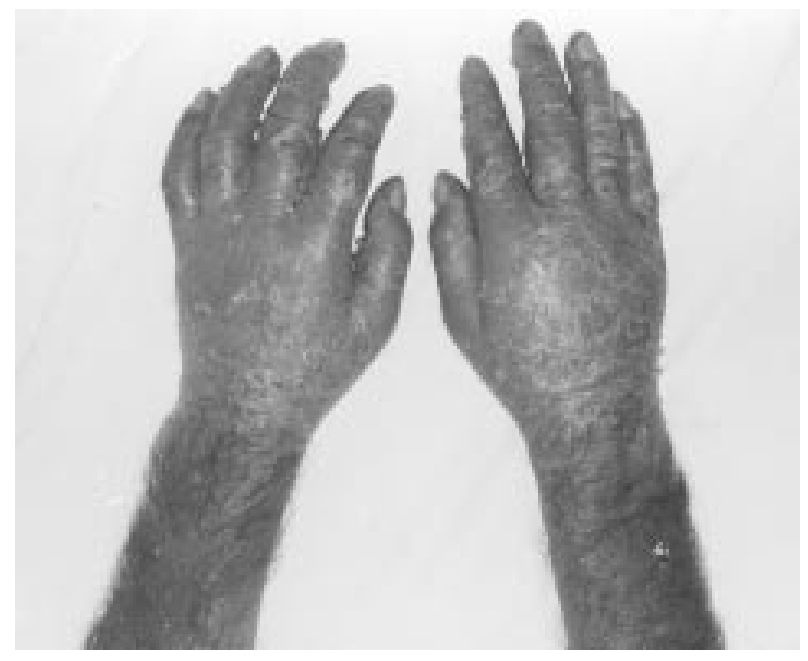

Figure 1 Dermatitis over dorsum of hands, sharply demarcated at wrist (reproduced with patient's permission).

consistent with subacute dermatitis. A diagnosis of pellagralike photosensitivity dermatitis caused by a combined deficiency of niacin, riboflavin, and other water soluble vitamins probably precipitated by haloperidol was thus considered. Haloperidol was stopped immediately, emollients were continued, and a multivitamin preparation containing niacin (100 mg) and riboflavin ( $10 \mathrm{mg}$ ), thrice daily, orally was started. The dermatitis and oral and eye symptoms resolved completely within the next five days. The next weekly dose of methotrexate was due after two days of resolution of this rash and led to a severe relapse of dermatitis within 24 hours but this time without oral and eye symptoms. As the relapse was of a different nature from that of the previous eruption and occurred despite being indoors and on a multivitamin supplement, the role of a methotrexate induced reactivation of photosensitivity was considered. The next weekly dose of methotrexate was withheld and multivitamin supplementation was also stopped. Dermatitis resolved completely within a week. After two weeks of resolution of the dermatitis, the patient was restarted on weekly methotrexate as before without any relapse over the next three months of follow up.

\section{DISCUSSION}

Photosensitivity is an adverse effect of many drugs, characteristically producing skin lesions in the areas exposed to light, which includes the face, " $V$ " area of the neck, extensor surfaces of forearms, and dorsa of hands with sparing of submental and retroauricular areas. Two major mechanisms mediating drug induced photosensitivity reactions are phototoxic and photoallergic responses. The phototoxic response resembles a primary irritant reaction and the lesions are erythematous and oedematous accompanied by a burning sensation. In contrast the photoallergic response mimics an 
ordinary allergic contact dermatitis and its morphological appearance is vesicular and eczematous with intense pruritus. Tetracyclines, phenothiazines, sulphonamides, non-steroidal anti-inflammatory drugs, amiodarone, psoralens, and coal tar derivatives are common causes of drug induced phototoxic reactions while halogenated salicylanilides, phenothiazines, benzodiazepines, sunscreen agents, and piroxicam are photoallergic drugs. Photosensitising agents may also result in an exacerbation or induction of a disease with a photosensitive component such as pellagra, in which a deficiency of niacin or tryptophan results in a deficiency of coenzymes that aid in repair of ultraviolet induced epidermal damage. ${ }^{7}$ Pellagra-like dermatitis has been associated with the use of drugs like isoniazid, 5-fluorouracil, 6-mercaptopurine, pyrazinamide, and hydantoins. $^{57}$

Photosensitivity is a well documented side effect of methotrexate and in a true photosensitivity reaction simultaneous exposure to both the drug and ultraviolet radiation results in enhancement of the radiation effect. False photosensitivity or recall or delayed reactivation phenomena is a rare adverse effect of methotrexate in which it reactivates an ultraviolet-B or radiation induced injury within one to four days of the institution of the drug. ${ }^{1-4}$ If the drug is introduced simultaneously or after four to seven days, it fails to evoke the reactivation of photosensitivity. ${ }^{3}$ Photosensitivity recall was first observed by Vogler et al in 1965 and was subsequently confirmed by others. $^{1-48}$ Although this recall phenomena is observed typically after an ultraviolet-B induced sunburn, methotrexate has rarely been observed to aggravate the photosensitivity induced by another drug like ciprofloxacin. ${ }^{4}$ Methotrexate has similarly been implicated in delayed reactivation of contact dermatitis and scabies. ${ }^{9}{ }^{10}$ A similar recall phenomenon has been reported with other cytotoxic drugs like Adriamycin, 5-fluorouracil, hydroxyurea, and vinblastine. ${ }^{11}$

The pathogenesis of this reactivation reaction, whether immunological or metabolic, is not well understood. Korossy and Hood have proposed that cells undergoing increased DNA, RNA, and protein synthesis during the epidermal hyperplasia after sunburn may be damaged by methotrexate. It may also inhibit local mononuclear cell responses to ultraviolet injury and thus augment local tissue damage. ${ }^{12}$

The initiation of pellagra-like dermatitis in this patient, whose psoriasis was otherwise well controlled on methotrexate treatment, was clearly associated with the institution of haloperidol. Its resolution with high dose niacin and multivitamins indicates that haloperidol probably interacted with tryptophan metabolism, which was overcome by a high dose of niacin. Reactivation of the dermatitis despite the patient being kept indoors because of hospitalisation and the failure of niacin to control the dermatitis suggests a different pathogenetic mechanism. The dermatitis persisted most probably because of the photosensitivity recall phenomena of methotrexate and subsided only when methotrexate was withdrawn. Since both methotrexate and haloperidol are not known to cause this kind of dermatitis when used alone, the

\section{Learning points}

- Patients on methotrexate should avoid sunburn or prolonged exposure to sun.

- Methotrexate should be avoided within one to four days of an acute sunburn reaction.

- Methotrexate can interact with "drugs causing photosensitivity" by reactivating their photosensitivity reaction

- Methotrexate can be reintroduced safely after two to three weeks of reactivation phenomena.

- A similar reactivation phenomena can be observed with Adriamycin, 5-fluorouracil, hydroxyurea, and vinblastine.

possibility of interaction between the two in precipitating this photosensitive dermatitis cannot be also excluded. With increased use of methotrexate in rheumatological and dermatological disorders, it is important to remember this recall photosensitivity of methotrexate, which can reactivate a drug induced photosensitivity.

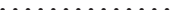

Authors' affiliations

G P Thami, S Kaur, A J Kanwar, Department of Dermatology and Venereology, Government Medical College and Hospital, Sector 32 B, Chandigarh 160047, India

Correspondence to: Dr Kanwar; goraya@glide.net.in

Submitted 4 April 2001

Accepted 21 August 200

\section{REFERENCES}

1 Neiman RA, Fye $\mathrm{KH}$. Methotrexate induced false photosensitivity reaction. J Rheumatol 1985;12:354-5.

2 Mallory SB, Berry DH. Severe reactivation of sunburn following methotrexate use. Pediatrics 1986:78:514-15.

3 Westwick TJ, Sheretz EF, McCarley T, et al. Delayed reactivation of sunburn by methotrexate: sparing of chronically sunexposed skin. Cutis 1987;39:49-51.

4 Nedorost ST, Dijkstra JWE, Handel DW. Drug induced photosensitivity reaction. Arch Dermatol 1989:125:433-4.

5 Hendricks WM. Pellagra and pellagra-like dermatoses: etiology differential diagnosis, dermatopathology and treatment. Semin Dermatol 1991;10:282-92.

6 Gerle B. Clinical observations of the side effects of haloperidol. Acta Psychiatr Scand 1964:40:65-76.

7 Gould JW, Mercurio MG, Elmets CA. Cutaneous photosensitivity diseases induced by exogenous agents. J Am Acad Dermatol 1995:33:551-73

8 Vogler WR, Huguley CM, Kerr W. Toxicity and anti tumour effect of divided doses of methotrexate. Arch Intern Med 1965;115:285-93.

9 Moller H. Reactivation of acute inflammation by methotrexate. J Invest Dermatol 1965;52:437-41.

10 Burrows D, Bridges JM, Morris TCM. Reactivation of scabies rash by methotrexate. Br J Dermatol 1975:93:219-21.

11 Moller H. Cytotoxic drugs and inflammation. Lancet 1967:ii:427

12 Korossy KS, Hood AF. Methotrexate reactivation of sunburn reaction. Arch Dermatol 1981;117:310-11. 